\title{
Data Acquisition and Readout System for the LUX Dark Matter Experiment
}

D.S. Akerib ${ }^{\mathrm{b}}$, X. Bai ${ }^{\mathrm{f}}$, S. Bedikian ${ }^{\mathrm{n}}$, E. Bernard ${ }^{\mathrm{n}}$, A. Bernstein ${ }^{\mathrm{e}}$, A. Bradley ${ }^{\mathrm{b}}$, S. B. Cahn ${ }^{\mathrm{n}}$, M. C. Carmona-Benitez ${ }^{\mathrm{b}}$,

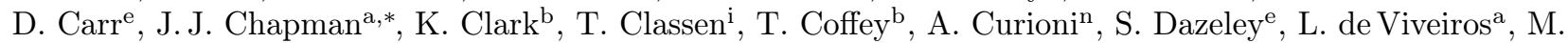

Dragowsky $^{\mathrm{b}}$, E. Druszkiewicz ${ }^{\mathrm{l}}$, C. H. Faham ${ }^{\mathrm{a}}$, S. Fiorucci ${ }^{\mathrm{a}}$, R. J. Gaitskell ${ }^{\mathrm{a}}$, K. R. Gibson ${ }^{\mathrm{b}}$, C. Hall ${ }^{\mathrm{k}}$, M. Hanhardt ${ }^{\mathrm{f}}$, B.

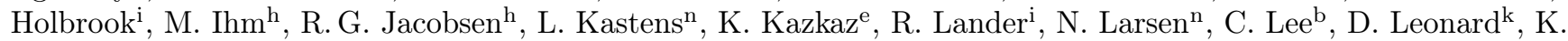

Lesko $^{d}$, A. Lyashenkon ${ }^{n}$ D. C. Malling ${ }^{\mathrm{a}}$, R. Mannino ${ }^{\mathrm{g}}$, D. N. McKinsey ${ }^{\mathrm{n}}$, D. Mei ${ }^{\mathrm{m}}$, J. Mock $^{\mathrm{i}}$, M. Morii $^{\mathrm{c}}, \mathrm{H}_{\text {. Nelson }}^{\mathrm{j}}$,

J. A. Nikkel ${ }^{\mathrm{n}}$, M. Pangilinan ${ }^{\mathrm{a}}$, P. Phelps ${ }^{\mathrm{b}}$, T. Shutt ${ }^{\mathrm{b}}$, W. Skulski ${ }^{1}$, P. Sorensen ${ }^{\mathrm{e}}$, J. Spaans ${ }^{\mathrm{m}}$, T. Stiegler ${ }^{\mathrm{g}}$, R. Svoboda ${ }^{\mathrm{i}}$,

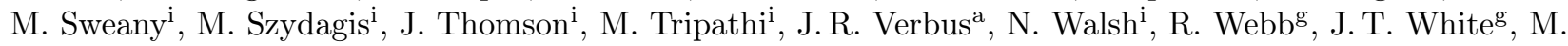

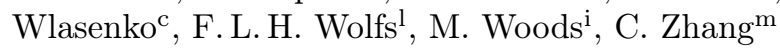

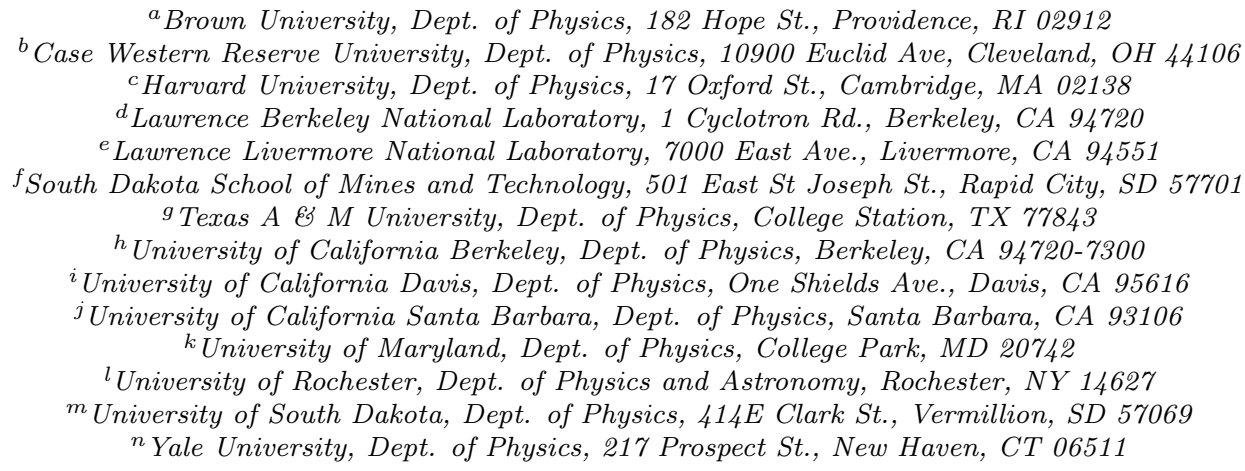

\section{Abstract}

LUX is a two-phase (liquid/gas) xenon time projection chamber designed to detect nuclear recoils from interactions with dark matter particles. Signals from the LUX detector are processed by custom-built analog electronics which provide properly shaped signals for the trigger and data acquisition (DAQ) systems. The DAQ is comprised of commercial digitizers with firmware customized for the LUX experiment. Data acquisition systems in rare-event searches must accommodate high rate and large dynamic range during precision calibrations involving radioactive sources, while also delivering low threshold for maximum sensitivity. The LUX DAQ meets these challenges using real-time baseline suppression that allows for a maximum event acquisition rate in excess of $1.5 \mathrm{kHz}$ with virtually no deadtime. This paper describes the LUX DAQ and the novel acquisition techniques employed in the LUX experiment.

\section{Introduction}

The LUX dark matter experiment is a two-phase xenon Time Projection Chamber (TPC). The goal of LUX is to detect energy depositions caused by galactic dark matter particles, known as Weakly Interacting Massive Particles or WIMPs, scattering off atomic nuclei [1, 2, 3, 4].

The site for LUX is the Davis Laboratory at the $4850 \mathrm{ft}$. level of the Sanford Underground Laboratory at the Homestake Mine in Lead, SD. The detector is housed in an $8 \mathrm{~m}$ diameter (300 tonne) water tank that acts as a background shield and muon veto. The LUX detector uses $350 \mathrm{~kg}$ of liquid xenon, $300 \mathrm{~kg}$ of which define the active region. This active region is observed by 122 Hamamatsu R8778 2 inch diameter photomultiplier tubes (PMTs). There are 61 PMTs in the top array and 61 in the bottom array.

*Corresponding Author: Jeremy_Chapman@brown.edu
The PMTs have an average quantum efficiency (QE) of $33 \pm 2.3 \%$ at the xenon scintillation wavelength of $175 \mathrm{~nm}$ [5]. The active region is walled by PTFE (polytetrafluoroethylene) reflector panels and is $59 \mathrm{~cm}$ in height $\times 49 \mathrm{~cm}$ in diameter [6, 7]. Particle interactions in the xenon create scintillation light and ionization electrons. Wire grids at the top and bottom of the liquid provide a uniform electric field in the active region. This electric field causes the electrons to drift from the interaction site towards the liquid surface. Additional grids at the surface provide a stronger electric field that extracts the electrons from the liquid and accelerates them in xenon gas, creating electroluminescence. The measurement of the drift time and the PMT hit pattern from the electro-luminescence allow for $3 \mathrm{D}$ localization of the original particle interaction.

The LUX data acquisition system (DAQ) is designed to simultaneously read out all 122 LUX PMTs, as well as the 20 PMTs observing the water shield, summed into 


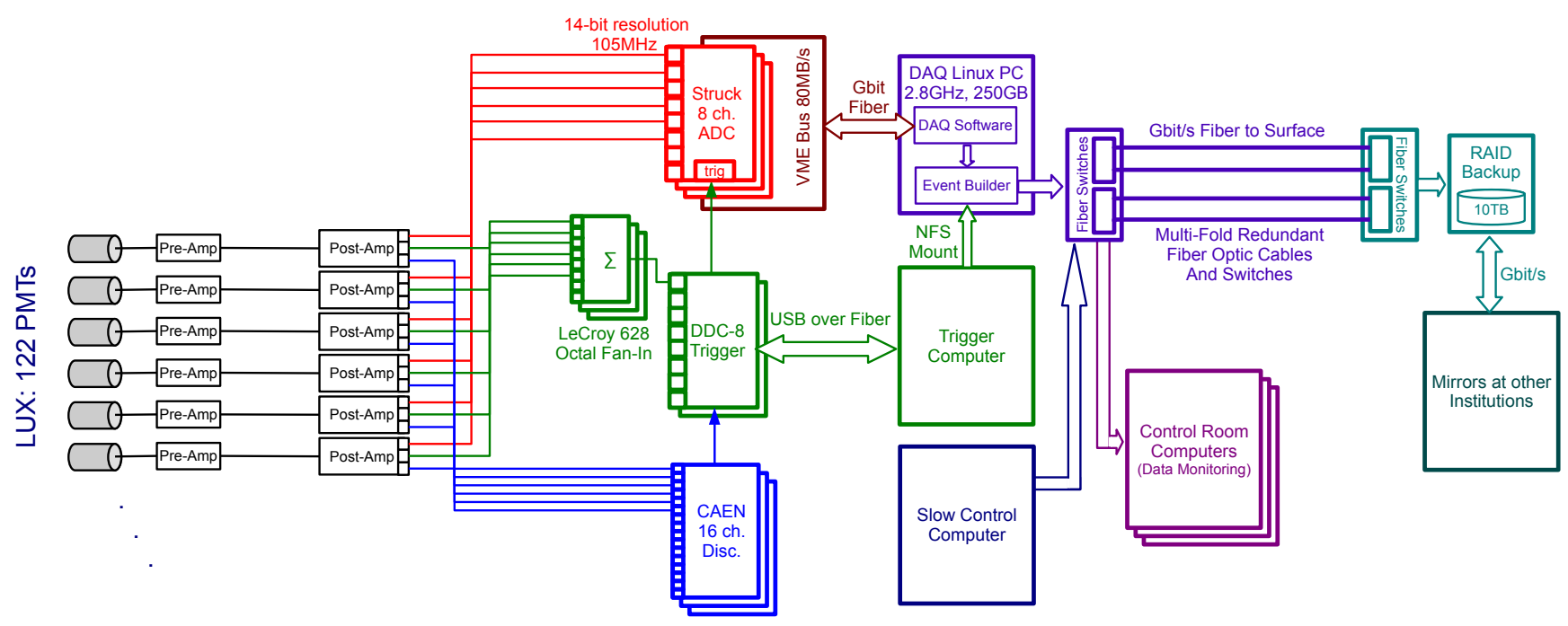

Figure 1: Data flow diagram of the LUX electronics and DAQ. Signals from the PMTs pass through air-side preamplifiers immediately after exiting the xenon space. A postamplifier further amplifies and shapes the signals. The postamplifier generates three outputs: one for the Struck ADCs, one for the DDC-8 digital trigger system, and one for the CAEN discriminators. The data from the Struck ADCs is read out from the VME bus to the DAQ computer, where it is sorted and prepared for analysis.

eight DAQ channels. The LUX readout is comprised of a custom-built analog electronics chain and triggering system, Struck fast 14-bit ADCs, and CAEN discriminators and scalers, as shown in Fig. 1] 8, 9]. The Struck digitizer firmware has been developed by the manufacturer in collaboration with LUX to perform real-time baseline suppression. This paper presents a description of the signals generated by interactions in the detector, the analog electronics chain designed to read out the PMT signals, and the data acquisition system. Data formatting and optimization techniques are also described.

\section{Signals}

There are two types of interactions in the detector: nuclear recoils (denoted by the $r$ subscript), which are interactions with the xenon nucleus, and electron recoils (denoted by the ee subscript), in which the particle interacts with the electrons. A dark matter WIMP particle is expected to produce a nuclear recoil in the detector, whereas gamma ray interactions will produce electron recoils [10]. A particle interaction in the detector is characterized by two scintillation signals. The first signal is the prompt scintillation of the interaction in the liquid xenon, and is known as the $\mathrm{S} 1$ pulse. For interactions in the xenon active region, S1 light is evenly distributed on the PMT arrays in $x y$. The majority of the light (80\%) falls on the bottom PMT array due to total internal reflection at the liquid surface. For an energy deposition of $2 \mathrm{keV}_{e e}$ in the center of the detector in the presence of a $1 \mathrm{kV} / \mathrm{cm}$ electric field, the S1 pulse will be comprised of about 50 photons [11]. The fundamental rise-time of the S1 light signal is limited by the response of the PMTs and is $\sim 6 \mathrm{~ns}$. The S1 signal decays exponentially with an effective time constant of $\tau=29 \mathrm{~ns}$ [12, 13. The S1 pulses are fully characterized by the DAQ in a time window of $<200 \mathrm{~ns}$. Monte Carlo simulations using Geant4 indicate that the S1 light collection in LUX will be 10 photoelectrons $/ \mathrm{keV}_{e e}$ (at an energy of $122 \mathrm{keV}_{e e}$ ) with no electric field applied, and assuming $33 \%$ PMT QE and 95\% PTFE reflectivity in liquid xenon. The S1 light collection becomes 5 photoelectrons $/ \mathrm{keV}_{e e}$ in the active region in the presence of an electric field of $1 \mathrm{kV} / \mathrm{cm}$.

The initial particle interaction also ionizes xenon atoms, releasing electrons at the interaction site. These electrons can recombine with their host atoms, producing recombination light which increases the size of the S1 signal. However, in LUX, the large electric field $(\gtrsim 0.1 \mathrm{kV} / \mathrm{cm})$ causes these electrons to drift from the interaction site towards the liquid surface before they can efficiently recombine [14. Grids, $0.5 \mathrm{~cm}$ above and below the liquid surface, generate strong electric fields of $5 \mathrm{kV} / \mathrm{cm}$ in the liquid and $10 \mathrm{kV} / \mathrm{cm}$ in the gas [6. This causes the electrons to leave the liquid and accelerate in the gas phase [15. The electrons produces further scintillation through electro-luminescence, proportional to the number of electrons extracted (see Fig. 20. This is known as the S2 pulse, and is about three orders of magnitude larger than the S1 pulse, depending on grid separation, applied field, and xenon gas pressure [15]. The ratio of S2 area to S1 area is used to discriminate between nuclear and electron recoils [3, 12]. LUX expects an S2 light yield of 35 photoelectrons per electron $\left(e^{-}\right)$extracted from the liquid and $45 e^{-} / \mathrm{keV}_{e e}$ for a gamma interaction in the detector at an energy of $10 \mathrm{keV}_{e e}$ [12, 13, 14, 16. This light yield ac- 
counts for geometric light collection in the gas phase where the S2 is generated, detection efficiency of the PMTs, and the number of scintillation photons produced per extracted electron. The S2 pulse waveform is gaussian-shaped when summed across all PMT channels. The S2 pulse width varies slightly as a function of depth because the cloud of electrons diffuses as it drifts towards the liquid surface [12, 13, 17]. The S2 is fully characterized by the DAQ in a time window of $\sim 3 \mu \mathrm{s}$. The S2 is spread over most PMTs, but shows significant localization in the top array above the electron extraction site. Monte Carlo simulations indicate that up to $22 \%$ of the S2 light is collected by a single PMT in the top array. This allows for $x y$ localization of the event with $\sigma=0.3 \mathrm{~cm}$ precision. In LUX, the time between S1 and S2 pulses, the electron drift time, is between 0 and $230 \mu \mathrm{s}$, given a field of $1 \mathrm{kV} / \mathrm{cm}$ and a maximum drift distance of $50 \mathrm{~cm} \mathrm{[6,} \mathrm{18].} \mathrm{This} \mathrm{drift} \mathrm{time} \mathrm{is} \mathrm{used} \mathrm{to}$ determine the $z$ coordinate of the interaction.

A typical event from LUX 0.1 is shown in Fig. 3. LUX 0.1 is a prototype detector using the LUX DAQ and electronics with four PMTs (3 top, 1 bottom) [7, 19]. The maximum drift time in LUX 0.1 is $25 \mu \mathrm{s}$. The detector is quiet between the S1 and S2 pulses, which are the most interesting features in the event.

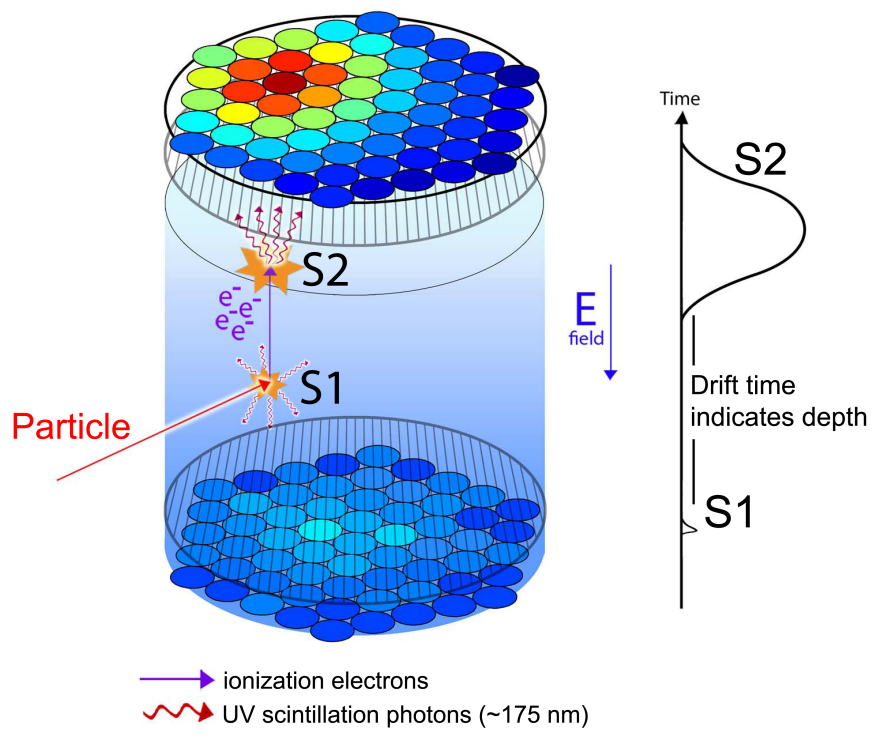

Figure 2: Diagram of a particle interaction in the LUX detector showing primary $(\mathrm{S} 1)$ and secondary (S2) scinitllation signals. The PMT hit pattern shown is generated by the $\mathrm{S} 2$ signal, where the gray scale from white (least) to black (most) indicates the number of photoelectrons detected by that PMT.

\section{Electronics Chain}

The LUX PMT readout is comprised of both analog and digital electronics. The analog electronics consists of two stages of amplifiers, designed to shape the PMT signals for use by the triggering and acquisition hardware.

\subsection{Digital Electronics}

The LUX DAQ uses 16 Struck 8-channel fast ADC modules, model number SIS3301, customized for LUX operation by Struck and Brown University. The Struck ADCs digitize at $105 \mathrm{MHz}$ with a resolution of 14 bits per channel and an intrinsic noise of $20 \mathrm{nV} / \sqrt{\mathrm{Hz}}$. Each input has a total voltage range of $-1900 \mathrm{mV}$ to $+100 \mathrm{mV}$. Note that the PMT signals are negative, but for the purposes of this paper the signals are described as positive. The Struck modules have $2 \times 128 \mathrm{k}$ sample dual memory banks that allow for acquisition on one bank while data is being downloaded from the other. The time to switch memory banks is $17 \mu \mathrm{s}$. Each board is connected to the VME bus, which is connected to the DAQ computer via a fiber optic Gbit connection, using Struck communication modules SIS3104 and SIS1100. The boards are capable of transferring data to the acquisition computer at a maximum VME download speed of $80 \mathrm{MB} / \mathrm{s}$ (2eVME protocol). Each board is controlled by four FPGAs, one per pair of channels, whose firmware was developed by Struck in collaboration with the Brown University group to operate in Pulse Only Digitization (POD) mode, described in section 4. Each Struck input has a $30 \mathrm{MHz}$ on-board single-pole anti-aliasing filter.

LUX is triggered using a digital triggering system, based on DDC-8DSP designed and built for LUX by collaborators at the University of Rochester and at SkuTek [20]. The DDC-8 digital triggering system digitizes the PMT signals at $64 \mathrm{MHz}$ with 14-bit resolution for processing by the on-board FPGA. Each DDC-8DSP input has a $24 \mathrm{MHz}$ on-board anti-aliasing filter. The DDC- 8 firmware has the ability to identify S1 and S2 pulses. This allows the DDC8 to trigger on S1s, S2s, or events with both an S1 and an $\mathrm{S} 2$. The DDC-8 trigger system has the ability to pre-select events of a given energy by measuring the size of the S1. The hit pattern of the S2 in the top PMT array, combined with the drift time between $\mathrm{S} 1$ and $\mathrm{S} 2$, allows the trigger system to pre-select events of a given position. The LUX trigger system and DDC-8DSP are discussed in [21, 22].

\subsection{Analog Electronics}

The PMT readout electronics are designed to maximize signal to noise in the dark matter search region of interest $\left(4-40 \mathrm{keV}_{r}\right)$ and to allow for high-energy calibrations. The first two stages (see Fig. 11 in the analog electronics chain, the preamplifier and the postamplifier, were designed and built by LUX collaborators at U. C. Davis, Lawrence Livermore National Laborabory, and Harvard University. The LUX readout requirements are based on the need for lowenergy calibrations in the dark matter search region, as well as high-energy calibrations $\left(\gtrsim 100 \mathrm{keV}_{e e}\right)$. These calibrations are necessary to examine the detector response to WIMP-like events, as well as to monitor the state of the 

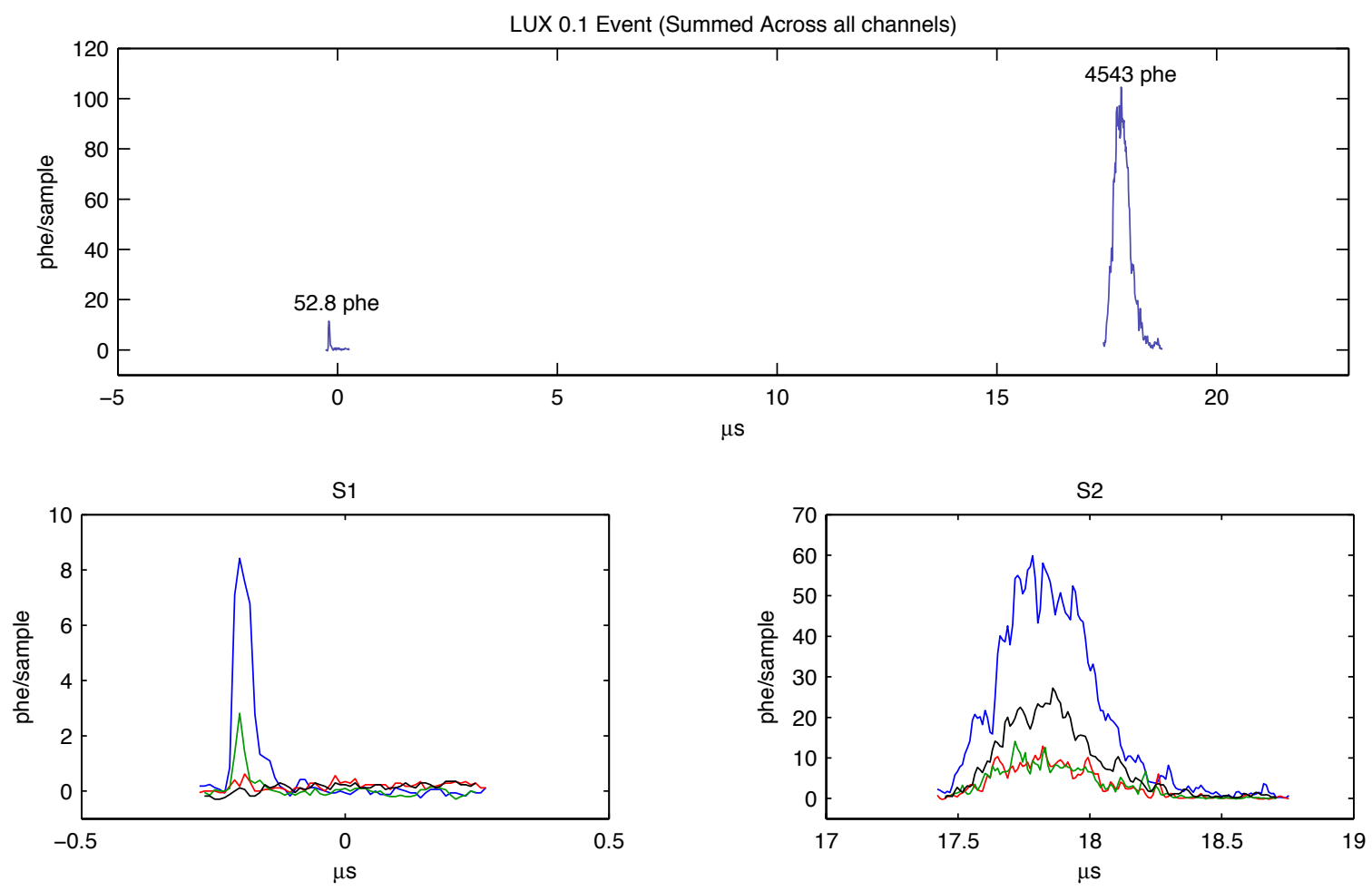

Figure 3: A $9.8 \mathrm{keV}_{e e}$ event from LUX 0.1 using Pulse Only Digitization (Sect. 4). (top) Sum of all four PMT channels. (bottom) The S1 and S2 signals in the individual PMTs. The number of samples before and after each pulse is selectable up to 24 and 31 respectively (9.5 ns/sample). Each PMT has a gain of $1 \cdot 10^{5}$.

detector systems. The former requires that the PMT and amplifier gains be equal to those used for WIMP searches. The latter allows for freedom in varying the PMT and amplifier gains. Divided into these two categories, there are four main detector calibrations:

(i) Electron lifetime (e-lifetime) calibrations are used to measure the purity of the xenon. Electro-negative impurities in the xenon absorb drifting electrons. This causes the size of the S2 to vary as a function of depth for a given interaction energy. The goal of xenon purification is to minimize this effect [12, 13, 19. The e-lifetime calibrations allow for a direct measurement of xenon purity and the depth dependence of the S2 signal. This can be achieved using gamma ray sources, such as ${ }^{137} \mathrm{Cs}$ or ${ }^{208} \mathrm{Tl}$, which produce gamma rays of high enough energies to penetrate into the center of the detector.

(ii) Nuclear and (iii) electron recoil calibrations are used to measure both relative scintillation efficiency and dark matter discrimination efficiency between the two event types. Electron recoil events are supplied using ${ }^{137} \mathrm{Cs}$, ${ }^{208} \mathrm{Tl},{ }^{133} \mathrm{Ba}$, and ${ }^{57} \mathrm{Co}$. Nuclear recoil events are supplied using ${ }^{252} \mathrm{Cf}$ and $\mathrm{AmBe}$ neutron sources. These calibrations must be made with PMT and amplifier gains equal to those used for dark matter searches.

(iv) Internal calibrations are used to unambiguously measure the uniformity of the detector. The two sources used for this are activated xenon and ${ }^{83 m} \mathrm{Kr} 23$.

During dark matter search mode it is desirable to max- imize trigger efficiency at the lowest energy threshold, and to completely cover the recoil energies expected for dark matter interactions. These requirements demand careful balancing between low threshold and large dynamic range.

The analog electronics chain starts inside the detector at the PMT base, which steps down the bias voltage for each dynode to produce electron multiplication. Custom Gore coaxial cables transmit the signal from the PMT base to the xenon space feedthrough [24. A single photoelectron pulse in this 10 meter long cable is attenuated to $75 \%$ of its original height while the pulse area is preserved. On the air side, a preamplifier is mounted to the signal feedthrough. The postamplifier is located near the Struck digitizers and the triggering hardware. Between the preamplifier and postamplifier another 10 meter long coaxial cable further attenuates the single photoelectron height to $83 \%$ of its original height, while the pulse area is preserved. In order to meet the aforementioned specifications, $\gtrsim 95 \%$ of single photoelectron pulses should be clearly resolved from $5 \sigma$ fluctuations in the baseline noise, and event energies up to $100 \mathrm{keV}_{e e}$ should not saturate any stage of the electronics. The R8778 PMTs are operated with a typical bias of $1.25 \pm 0.1 \mathrm{kV}$ to produce a of gain $3.3 \cdot 10^{6}$. Higher energy calibrations that will saturate the PMTs or electronics at this bias can be done by lowering either the PMT or the amplifier gains. At a gain of $3.3 \cdot 10^{6}$ a single photoelectron generates a pulse area of $13.3 \mathrm{mV} \cdot \mathrm{ns}$ at the output of the PMT base with $50 \Omega$ 
termination. The single photoelectron peak resolution is $\sigma=37 \%$ on average 25 . The preamplifier has a gain of $\times 5$, with an in-line switchable attenuator of $\times 1 / 10$, for an optional total gain of $\times 0.5$. The postamplifier has three outputs: one for the Struck digitizers, one for the DDC8 trigger system, and a third, for the discriminator and scaler modules. The postamplifier is an integrating amplifier, designed for an output bandwidth of $30 \mathrm{MHz}$, in order to match the input bandwidth of the Struck and the DDC-8 digitizers. The postamplifier is designed to give a gain in pulse area of $\times 1.5$ for the Struck digitizers and $\times 2.8$ for the DDC- 8 trigger system. The output for the discriminators has a gain of $\times 18$. Each of these outputs has optional $\times 0.5$ attenuators. The Struck and the DDC8 digitizers each have bandwidth limiting filters at their inputs with cutoff frequencies of $30 \mathrm{MHz}$ and $24 \mathrm{MHz}$, respectively. Each PMT channel is digitized by one Struck channel. The analog sum of eight PMT channels from the DDC- 8 output of the postamplifier is generated using LeCroy 628 octal fan-in modules and sent to one channel of the DDC-8 trigger system. This is done in order to reduce the cost of the trigger system. The trigger system is discribed in more detail in Refs. [21, 22].

\subsubsection{Dynamic Range:}

A design goal of the DAQ and electronics is that the maximum measureable energy deposition in the detector be limited by the PMTs and not by the electronics chain. The R8778 PMTs become $2 \%$ nonlinear with an instantaneous current draw of $13 \mathrm{~mA}$ at the anode [5, 25]. Simulations predict that a PMT in the top array, located directly above the electron extraction site, will see at most $22 \%$ of the total S2 light. The maximum area of the S2 signal, combined across all channels without saturating any PMT, is $2 \cdot 10^{5}$ photoelectrons. This corresponds to a maximum event energy of $\sim 120 \mathrm{keV}_{e e}$, well above the desired calibration range of $100 \mathrm{keV}_{e e}$.

At the Struck output of the postamplifier, a single photoelectron has a height of $4 \mathrm{mV}$ and an area of $100 \mathrm{mV} \cdot \mathrm{ns}$, assuming a PMT gain of $3.3 \cdot 10^{6}$. The S1 light is typically split; $20 \%$ on the top PMT array and $80 \%$ on the bottom. The S1 signal on the bottom PMT array is evenly spread across all 61 channels. With a maximum input voltage on the Struck ADC of $1900 \mathrm{mV}$, the largest non-saturating S1 pulse has an area of $4.2 \cdot 10^{4}$ photoelectrons. This corresponds to a maximum energy deposition of $8.4 \mathrm{MeV}_{e e}$, assuming a light collection efficiency of 5 photoelectrons $/ \mathrm{keV}_{e e}$ in the presence of an electric field $\gtrsim 0.5 \mathrm{kV} / \mathrm{cm}$. The maximum area of the $\mathrm{S} 2$ signal combined across all channels, without saturating the Struck ADC in any single channel, is $1.1 \cdot 10^{6}$ photoelectrons. This corresponds to a maximum event energy of $715 \mathrm{keV}_{e e}$.

\subsubsection{Threshold:}

The Struck SIS3301 is a 14-bit digitizer with a total voltage range of $2 \mathrm{~V}$. A threshold of 13 ADC counts is below the height of $95 \%$ of single photoelectron signals.
The total noise from the electronics chain and ADC, as measured at the Struck input, is $155 \mu \mathrm{V}_{R M S}$. A $5 \sigma$ upward fluctuation of this noise is $6.5 \mathrm{ADC}$ counts. This is comfortably below the threshold of 13 ADC counts.

\section{Pulse Only Digitization}

Pulse Only Digitization (POD) is an acquisition mode optimized for signals that are dominated by long periods of baseline, interspersed with short pulses. The purpose of POD mode is to acquire only the pulses in the data stream, while suppressing the baseline. POD operates by saving to memory only signals that rise above a threshold. This improves the DAQ livetime by preventing the baseline between pulses from dominating the data collected. An example of an event with and without POD mode from LUX 0.1 is shown in Fig. 4.

POD mode operates on channel pairs since the Struck memory banks are organized per pair of channels. When one channel of a pair goes above threshold and saves data to memory, the data from the partner channel is recorded as well, even if the signal on that channel did not go above threshold.

Each pulse is recorded in the Struck memory with a header. This header has a unique identifier to signify the beginning of a pulse in memory. It also contains the 48-bit timestamp which is relative to the start of the acquisition, the number of samples in the pulse, the value of the baseline in ADC counts when the pulse was recorded, and a flag indicating which channel(s) in the pair went above threshold. A separate set of registers contain the timestamps and memory addresses of the first 256 pulses saved to memory. This facilitates download of only a portion of the data from the memory bank. This is used in singleevent mode to download only the pulses that are associated with the event trigger, as discussed in Sect. 6 .

Pulse acquisition on each channel is controlled by three independent thresholds, set prior to the start of the acquisition. Each threshold is illustrated in Fig. 5. The pulse detect threshold indicates the start of the pulse; pulse end threshold indicates the end of the pulse; and the pulse overshoot threshold can be used to record portions of the pulse that overshoot the baseline. Pulse overshoot occurs when the signal crosses zero for a short time before returning to the baseline. This feature ensures that bipolar noise glitches are fully characterized by the DAQ for analysis. These thresholds can be independently specified for each channel.

Each digitized sample is placed in a rolling buffer of 24 samples. When a pulse is detected, up to 24 samples of pretrigger can be recorded with it. Each pulse can have as many as 31 posttrigger samples recorded as well. An example of a single photoelectron pulse, digitized in LUX with POD mode, is shown in Fig. 6. The pulse detect threshold is set at 13 ADC counts and corresponds to a single photoelectron detection efficiency of $95 \%$. The $5 \sigma$ upward fluctuation in the baseline noise is also indicated. 

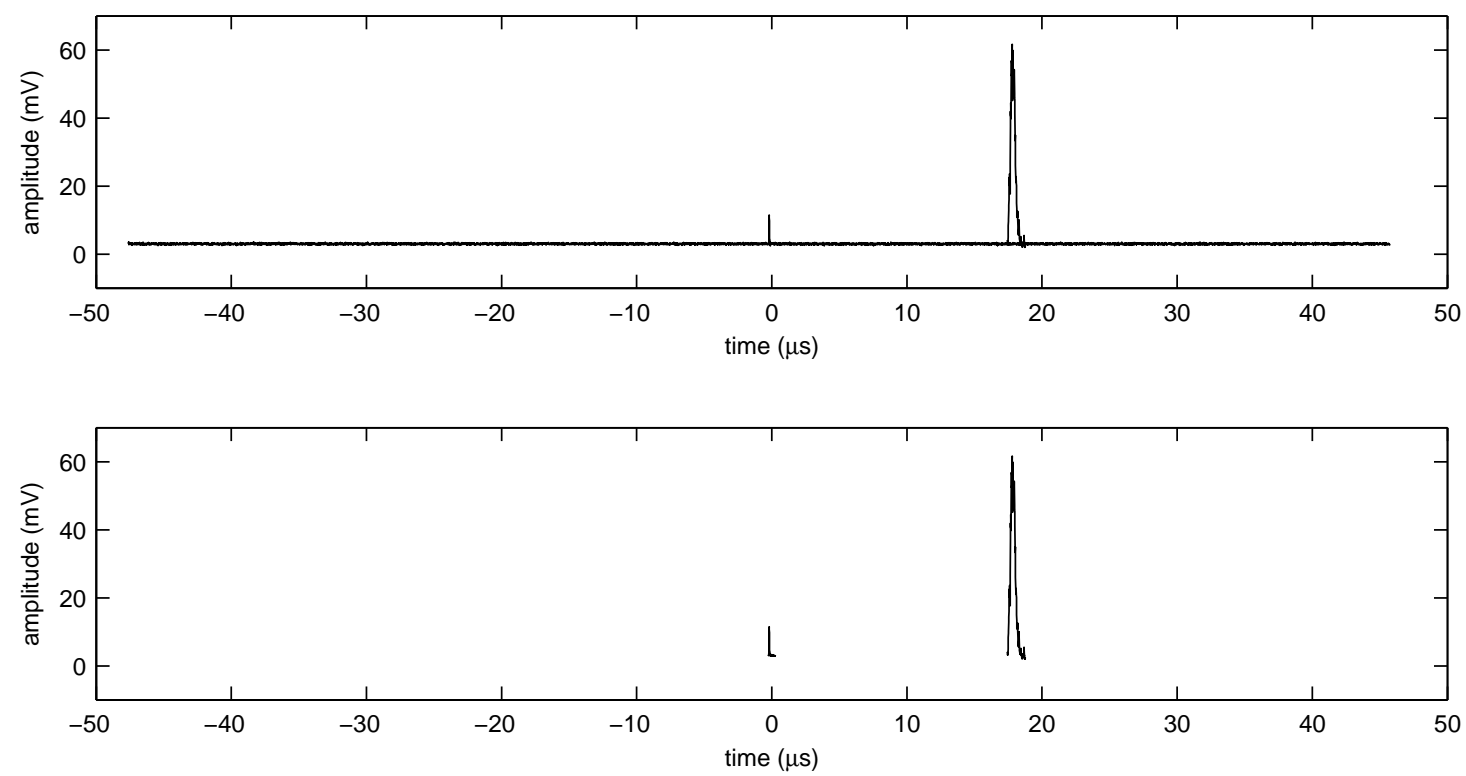

Figure 4: Sample event from the bottom PMT in LUX 0.1 digitized with (bottom) and without (top) Pulse Only Digitization (POD).

At this threshold, with the maximum pretrigger and posttrigger samples, the baseline suppression of the DAQ in POD mode is $99.99 \%$. This is measured by keeping the PMT bias at $-100 \mathrm{~V}$, below the threshold necessary to genrate detectable signals, and counting the number of samples recorded per second of acquisition. The only samples recorded are those associated with noise glitches. This is addressed with the Valid Pulse Trigger Gate, described in Sect. 4.1. POD mode gives a factor of $\times 1 / 50$ reduction in storage size for a typical event over the full drift length of LUX, compared to non-POD mode acquisition.

The acquisition is designed to allow for slow changes in the baseline caused by low-frequency noise, such as $60 \mathrm{~Hz}$ pickup. The baseline of each channel is determined by a rolling average of $16,32,64$, or 128 samples. This number specified by the user at the start of the acquisition. 128 samples was chosen for LUX, based on the noise profile and the PMT signals. The pulse detection thresholds are measured relative to this baseline. This rolling average is calculated until the pulse detect threshold is crossed, at which point its value is recorded in the pulse header. By freezing the calculated baseline at the beginning of the pulse, a large signal will not artificially raise the rolling average, which would in turn raise the pulse detection threshold. The rolling average baseline calculation is restarted at the end of the posttrigger samples.

\subsection{Valid Pulse Trigger Gate}

Whenever the memory bank on one Struck digitizer fills up, all Struck modules switch memory banks. This takes up to $290 \mu \mathrm{s}$. This deadtime is not significant if the pulse rate is dominated by $\mathrm{S} 1$ and $\mathrm{S} 2$ light from particle interactions in the detector. However, if one channel is

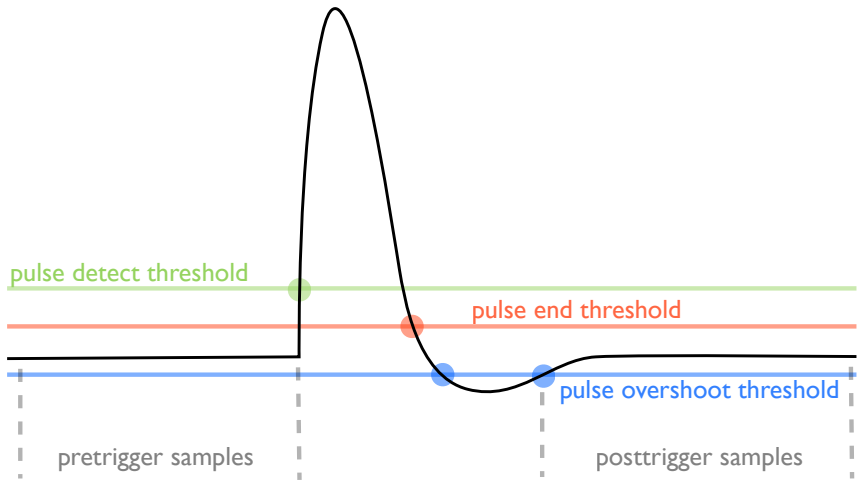

Figure 5: Illustration of Pulse Only Digitization (POD) mode on an arbitrary signal (see Sect. 4).

dominated by noise, it can introduce significant deadtime by forcing the Struck modules to switch memory banks frequently. This noise may not be coincident across more than one channel. The Valid Pulse Trigger Gate (VPTG) eliminates this problem. The VPTG ensures that only those pulses that are coincident in more than one channel are recorded to memory. It is expected that no S1 or S2 pulses will occur in only one channel, whereas many types of noise glitches will.

The VPTG is implemented using CAEN V814 Discriminators with majority coincidence logic. A majority decision is made in about $2 \mu \mathrm{s}$, limited by the width of the $\mathrm{S} 2$ pulse. The gate will only turn $\mathrm{ON}$ when more than one channel detects a signal. After the ADC, the signal is passed through a variable delay buffer between 0 and 254 samples $(0-2.42 \mu \mathrm{s})$. This is required because the Valid Pulse Trigger Gate is delayed by as much as $2 \mu$ s relative 


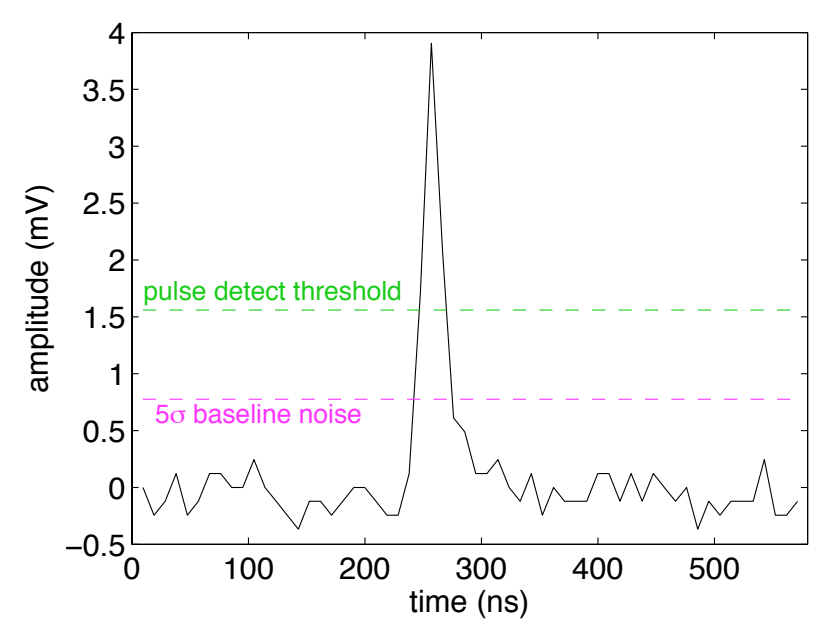

Figure 6: Single photoelectron pulse from a PMT in LUX detected with POD mode (see text for details). The pulse detect threshold corresponding to $95 \%$ of single photoelectrons, and $5 \sigma$ upward fluctuation in the baseline noise are also indicated.

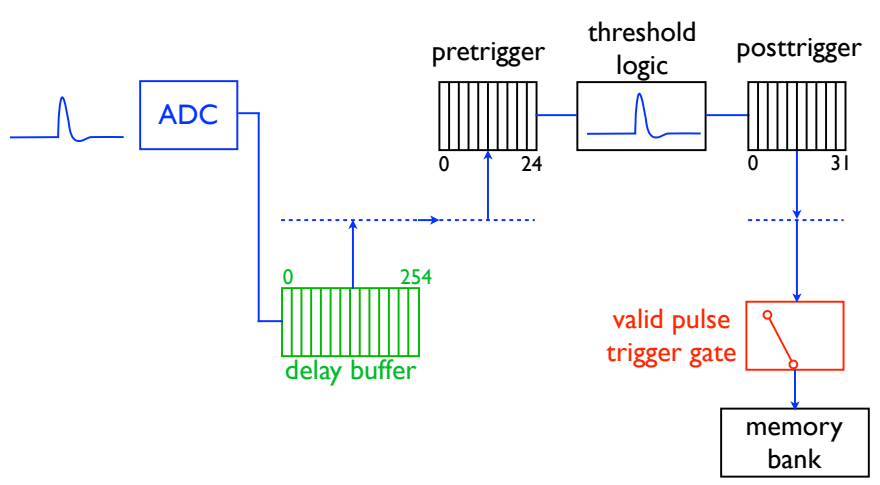

Figure 7: Diagram of Valid Pulse Trigger Gate (VPTG) and delay buffer implementation in the Struck ADC.

to the actual signal. After exiting the delay buffer, the signal enters the variable length pretrigger region which can be between 0 and 24 samples $(0-240 \mathrm{~ns})$. The signal then passes through the threshold logic, where the beginning and end of the pulse are determined. Between 0 and 31 posttrigger samples $(0-310 \mathrm{~ns})$ are recorded after the end of the pulse. If the VPTG is ON, then the data between the start of the pretrigger and the end of the posttrigger is stored in the memory bank. If the VPTG is OFF, then the pulse is discarded. This is summarized in Fig. 7. Using this delay buffer and the VPTG, it is possible to synchronize the threshold logic of the Struck with the coincidence decision made by the discriminators.

\section{DAQ Control}

Each Struck digitizer has four programmable LEMO inputs and four outputs. These are listed in Table 1. The clock and timestamp start time of all Struck boards are

\begin{tabular}{|c|c|c|}
\hline & Inputs & Outputs \\
\hline \hline 1 & $\begin{array}{c}\text { Clear Timestamp or } \\
\text { VPTG }\end{array}$ & Sample Gate \\
\hline 2 & Stop & $\begin{array}{c}\text { Acquisition Stopped or } \\
\text { Threshold Crossed }\end{array}$ \\
\hline 3 & Start & End Address Threshold \\
\hline 4 & Clock In & Clock Out \\
\hline
\end{tabular}

Table 1: Function of Struck SIS3301 control inputs and outputs.

synchronized using the control inputs/outputs. One module serves as the master, using its own internal clock. Its clock is then provided to all of the other modules through Clock Out via Philips 740 linear quad fan-out NIM modules. All other Struck boards are in slave clock mode, and use the clock provided on their Clock In. This ensures that the clocks are synchronized across all boards during acquisition. The Clear Timestamp input is used to synchronize the start of acquisition across all boards. This signal is generated by the master and provided to the slaves via LeCroy 128L octal fan-out NIM modules. After acquisition is started, the function of input 1 is changed via software to the VPTG, which is discussed in Sect. 4.1. Acquisition on each board can be started and stopped sequentially via software, or simultaneously via the Start and Stop inputs. The End Address Threshold output indicates when the board has reached the end of one of its memory banks and has stopped sampling. Output 2 indicates either that acquisition has stopped (by software or Stop input) or that the pulse detection threshold has been crossed and the Struck digitizer is recording a pulse. The Sample Gate can be used to indicate the livetime of each board. The gate is ON when the board is sampling and OFF otherwise. The flexibility of the control inputs/outputs allows for a robust characterization of the DAQ system.

Data acquisition is initiated by starting all boards via software and synchronizing their timestamps. When acquisition is stopped on any one board, indicated by control output 2, all boards are stopped simultaneously via control input 2. When this happens, the memory banks are switched in software and the master is started. At this point the sampling gate (output 1) turns ON and all other boards are started simultaneously via control input 3. This minimizes the deadtime of the DAQ system by starting and stopping all boards simultaneously.

\subsection{Software}

The acquisition software is used to start and stop acquisition, download and write data to disk, and manage the switching of memory banks. The acquisition settings are displayed in a graphical user interface and stored in a MySQL database. The settings are also copied to the header of each data file as it is written, allowing for easy reference when loading files. The settings specified include the length of the acquisition, which channels are enabled, 


\begin{tabular}{|c|c|c|c|}
\hline Mode & Max. Rate & Description & Benefit \\
\hline \hline Multi-event & $\begin{array}{c}\text { 1.5 kHz raw } \\
\text { detector } \\
\text { event rate }\end{array}$ & $\begin{array}{c}\text { Acquire all pulses, sorting } \\
\text { triggered events offline in } \\
\text { software. }\end{array}$ & $\begin{array}{c}\text { Debug triggering system; } \\
\text { examine detector background } \\
\text { offline, irrespective of trigger. }\end{array}$ \\
\hline Single-event & $\begin{array}{c}300 \mathrm{~Hz} \\
\text { triggered } \\
\text { events }\end{array}$ & $\begin{array}{c}\text { Cycle acquisition memory } \\
\text { banks after every trigger to } \\
\text { download only pulses in } \\
\text { triggered events. }\end{array}$ & $\begin{array}{c}\text { Acquire only triggered events, } \\
\text { making full use of digital trigger. }\end{array}$ \\
\hline
\end{tabular}

Table 2: Summary of acquisition modes and their uses.

threshold levels, the number of pretrigger and posttrigger samples, and the number of samples to average over when calculating the rolling baseline. The software itself is written in $\mathrm{C}$ and runs on a Linux computer with Struck drivers for communication with the VME crate. The data is written to disk in a binary format similar to that read out from the Struck memory banks in order to reduce deadtime due to file writing. The data is further sorted by the Event Builder, as discussed in Sect. 7 .

\subsection{Livetime}

When the DAQ software initiates an acquisition, the first memory bank on each Struck digitizer board is enabled. The initialization timestamp is recorded for each board whenever a memory bank is enabled. The acquisition is considered live when all boards are acquiring. When the acquisition is stopped, the timestamp indicating when the first board stopped acquiring is recorded. The acquisition is considered dead when any one board is not acquiring. These values are recorded in the header of the data file.

\section{Acquisition Strategies}

The LUX DAQ operates in either calibration or WIMP search mode. These modes have very different requirements. Calibration sources are chosen so that the total event rate in the detector does not exceed $200 \mathrm{~Hz}$. This rate keeps the percentage of events that overlap in time below $10 \%$, for a maximum drift time of $230 \mu \mathrm{s}$. In WIMP search mode, the expected background rate in the detector is $1.2 \mathrm{~Hz}$ [13.

The flexibility of the DAQ design allows for two acquisition strategies to be used: multi-event mode and singleevent mode. They are summarized in Table 2 and described in this section.

\subsection{Multi-event Mode}

In multi-event mode, the DAQ records all pulses from the PMTs, regardless of the trigger condition. Trigger information is recorded as part of the data stream and used to identify events in software. When the memory bank of one Struck digitizer becomes full, all of the data from all Struck digitizers is downloaded to the DAQ computer. The maximum acquisition rate for multi-event mode is $1.5 \mathrm{kHz}$ without any deadtime due to data transfer from the digitizers. Multi-event mode also saves pulses in the detector that are not necessarily part of a triggered event. This allows for the trigger and DAQ systems to be debugged and for all pulses in the detector to be more closely examined.

\subsection{Single-event Mode}

In single-event mode, the DAQ alternates between Struck memory banks for every trigger. Only the pulses that belong to the event that was flagged by the trigger system are downloaded to the DAQ computer. This fully utilizes the DDC-8 trigger to pre-select desired events. This keeps unwanted events from congesting the data stream and analysis. Because the DAQ needs to cycle Struck memory banks for each event, the maximum acquisition rate without deadtime is limited to about $300 \mathrm{~Hz}$. Note that this is the rate of events flagged by the trigger system, not the rate of all particle interactions in the detector. The latter is limited to $200 \mathrm{~Hz}$ by event overlap constraints. The ability to pre-select events using the DDC- 8 makes this mode ideal for acquiring calibration data because it is desirable to record only events with certain energies or at certain locations within the active region.

\section{Data Formatting and Reduction}

When the raw data from the Struck digitizers is written to file, it is sorted by channel and then by pulse. The sorting is chronological, but shows no relation to the actual event triggers. The Event Builder uses the trigger information to sort the data by event and then by channel, and finally by pulse. An index file is also recorded to easily relate the location of each event in the event files. The Event Builder serves three purposes: (1) it sorts the data so that it is easily parsed for event traces; (2) it incorporates traces from the water shield PMTs as well as information from the trigger system into the data stream; and, (3) it discards any pulses that were recorded in the file that do not belong to an event (lying in the event window determined by the trigger mode). Item (3) is particularly useful in multi-event mode where all pulses recorded by the Struck digitizers are written to file, independent of the event triggers. The Event Builder is written in $\mathrm{C}$ and runs 
in parallel to the acquisition on the DAQ computer. The Event Builder writes one output file per raw data file, and one index file per acquisition. The number of events in a raw file can be specified at the start of the acquisition to keep size and number of files from becoming cumbersome. Acquisition settings, Event Builder settings, trigger settings, and water tank readout settings are all recorded in the header of each file.

\section{Conclusion}

The LUX data acquisition system is finely tuned and optimized for the LUX dark matter experiment. This is achieved mainly by using custom-built analog electronics and custom digitization firmware. The dynamic range is optimized for performance at low energies for dark matter searches, as well as for high-energy calibrations. The noise level is limited such that $95 \%$ of single photoelectrons are clearly visible above a $5 \sigma$ fluctuation in the baseline noise. A novel real-time baseline suppression technique has been employed in the digitizers to provide a factor of $\times 1 / 50$ reduction in storage size. The maximum event rate achieved by the DAQ, without incurring significant deadtime, is $1.5 \mathrm{kHz}$. This is well above what is needed for calibrations and WIMP searches. While being finely tuned, the LUX DAQ maintains flexibility in acquisition techniques and strategies.

\section{Acknowledgments}

The authors would like to recognize the assistance of Matthias Kirsch and Tino Haeupke at Struck Innovative Systeme for their work on the ADC firmware. This work was partially supported by the U.S. Department of Energy (DOE) grants DE-FG02-08ER41549, DE-FG02-91ER40688, DE-FG02-95ER40917, DE-FG02-91ER40674, DE-FG0211ER41738, DE-FG02-11ER41751, DE-AC52-07NA27344, the U.S. National Science Foundation grants PHYS-0750671, PHY-0801536, PHY-1004661, PHY-1102470, PHY-1003660, the Research Corporation grant RA0350, the Center for Ultra-low Background Experiments at DUSEL (CUBED), and the South Dakota School of Mines and Technology (SDSMT). We gratefully acknowledge the logistical and technical support and the access to laboratory infrastructure provided to us by the Sanford Underground Research Facility (SURF) and its personnel at Lead, South Dakota.
[3] XENON Collaboration, J. Angle et al., "First Results from the XENON10 Dark Matter Experiment at the Gran Sasso National Laboratory," Phys. Rev. Lett. 100 (2008) 021303. arXiv:0706.0039 [astro-ph]

[4] E. Aprile, et al., "The XENON dark matter search experiment," New Astronomy Reviews 49 (2005) no. 2-6, 289 295. [link] Sources and Detection of Dark Matter and Dark Energy in the Universe.

[5] Hamamatsu Photonics Co. [link] http://www.hamamatsu.com.

[6] D. N. McKinsey, et al., "The LUX Dark Matter Search," in TAUP. Gran Sasso National Laboratory, Italy, 2009.

[7] S. Fiorucci, et al., "Status Of The LUX Dark Matter Search," in SUSY09. Boston, MA, 2009.

[8] Struck Innovative Systems. [link] http://www.struck.de/.

[9] CAEN SpA. [link] http://www.caen.it/.

[10] R. J. Gaitskell, "Direct Detection of Dark Matter," Ann. Rev. Nucl. Part. Sci. 54 (2004) 315-359. [doi]

[11] T. Doke, et al., "Absolute Scintillation Yields in Liquid Argon and Xenon for Various Particles," Japanese Journal of Applied Physics 41 (2002) no. Part 1, No. 3A, 1538-1545. [link]

[12] P. Sorensen, A Position-Sensitive Liquid Xenon Time-Projection Chamber for Direct Detection of Dark Matter: The XENON10 Experiment. PhD thesis, Brown University, July, 2008.

[13] L. de Viveiros, Optimization of Signal versus Background in Liquid Xe Detectors used for Dark Matter Direct Detection Experiments. PhD thesis, Brown University, November, 2009.

[14] C. E. Dahl, The physics of background discrimination in liquid xenon, and first results from Xenon10 in the hunt for WIMP dark matter. PhD thesis, Princeton University, September, 2009.

[15] A. I. Bolozdynya, "Two-phase emission detectors and their applications," Nuclear Instruments and Methods in Physics Research Section A: Accelerators, Spectrometers, Detectors and Associated Equipment 422 (1999) no. 1-3, 314 - 320. [link].

[16] T. Takahashi, et al., "Average energy expended per ion pair in liquid xenon," Phys. Rev. A 12 (Nov, 1975) 1771-1775. [doi]

[17] P. Sorensen, "nisotropic diffusion of electrons in liquid xenon with application to improving the sensitivity of direct dark matter searches," Nucl. Instr. Meth. A 635 (2011) 41-43.

[18] L. S. Miller, S. Howe, and W. E. Spear, "Charge Transport in Solid and Liquid Ar, Kr, and Xe,"Phys. Rev. 166 (Feb, 1968) 871-878. [doi]

[19] D. S. Akerib, et al., "The LUX 0.1 Detector," To be submitted to Nucl. Inst. Meth. A .

[20] SkuTek. [link] http://www.skutek.com.

[21] D. S. Akerib, et al., "Digital Triggering System for the LUX Dark Matter Experiment," To be submitted to IEEE Trans. Nucl. Sci. .

[22] W. Skulski, et al., "DDC-8DSP," To be submitted to Nucl. Inst. Meth. A

[23] L. W. Kastens, et al., "Calibration of a Liquid Xenon Detector with Kr-83m,"Physical Review C (Nuclear Physics) (Oct., 2009) . arXiv:0905.1766.

[24] W. L. Gore \& Associates, Inc. [link] http://www.gore.com.

[25] D. S. Akerib, et al., "The LUX Photomultiplier Tube Program," To be submitted to Nucl. Inst. Meth. A.

\section{References}

[1] D. Akimov, et al., "The ZEPLIN-III dark matter detector: Instrument design, manufacture and commissioning," Astroparticle Physics 27 (2007) no. 1, 46 - 60. [link]

[2] G. Alner, et al., "First limits on WIMP nuclear recoil signals in ZEPLIN-II: A two-phase xenon detector for dark matter detection," Astroparticle Physics 28 (2007) no. 3, $287-302$. [link]. 